\title{
A Digital Image Watermarking Algorithm Based on DWT DCT and SVD
}

\author{
Md Saiful Islam and Ui Pil Chong
}

\begin{abstract}
Digital watermarking techniques have been developed to protect the copyright of multimedia objects such as text, audio, video, etc. In this paper, we propose a new digital watermarking algorithm with gray image based on discrete wavelet transform (DWT), 2 dimensions discrete cosine transform (DCT) and singular value decomposition (SVD) for robust watermarking of digital images in order to protect digital media copyright efficiently. One of the major advantages of the proposed scheme is the robustness of the technique on wide set of attacks. Experimental results confirm that the proposed scheme provides good image quality of watermarked images.
\end{abstract}

Index Terms-Digital image watermarking, DWT, DCT PSNR, SVD.

\section{INTRODUCTION}

In the present globalization, the availability of the Internet and various image processing tools opens up to a greater degree, the possibility of downloading an image from the Internet, Manipulating it without the permission of the rightful owner. For reason such as this and many others, image authentication has become not only an active but also vital research area. Embedding watermarks [1]-[4] in both signals and images can cause distortion in them.

In general, a successful watermarking scheme should satisfy the following fundamental requirements.

1) Imperceptibility: the perceptual difference between the watermarked and the original documents should be unnoticeable to the human eye, i.e. watermarks should not interfere with the media being protected.

2) Trustworthiness [5]-[8]: a satisfactory watermarking scheme should also guarantee that it is impossible to generate forged watermarks and should provide trustworthy proof to protect the lawful ownership.

3) Robustness [9]-[12]: an unauthorized person should not be able to destroy the watermark without also making the document useless, i.e., watermarks should be robust to signal processing and intentional attacks. In particular, after common signal processing operations have been applied to the watermarked image like filtering, re-sampling, cropping, scaling, digital-to-analog, analog-to-digital conversions, compression, geometric transformation, rotation, etc., they should still be detectable.

Generally, watermarking can be classified into two groups: spatial domain methods and transform domain methods. In

Manuscript received January 20, 2014; revised March 15, 2014. This work was supported in part by the University of Ulsan.

The authors are with the University of Ulsan, Ulsan, South Korea (e-mail: saifu105eee@yahoo.com,upchong@ulsan.ac.kr). spatial domain approaches, the watermark is embedded directly to the pixel locations [13], [14]. Embedding the watermark in the spatial domain is the direct method. It has various advantages like less computational cost, high capacity, more perceptual quality but less robust and it mainly suits for authentication applications. In transform domain approaches, a mathematical transform is applied to the original image to embed watermark into the transform coefficients, then apply inverse transform to get the embedded image. It has more robust, less control of perceptual quality and mainly suits for copyright application. The most frequent used methods are discrete cosine transform (DCT) domain [15], [16], discrete wavelet transform (DWT) domain [17], singular value decomposition (SVD) domain [18]. They now come into more widespread used as they always have good robustness to common image processing.

In this paper a DCT DWT SVD based blind watermarking technique has been used for embedding watermark. A new watermarking algorithm based on DWT, DCT and SVD, for digital image indicate that this algorithm combines the advantages of these three transforms. It can proof the imperceptibility and robustness very well. Moreover, the algorithm is robust to the common image process such as Filtering, Gaussian noise, Rotation and Salt and Pepper.

The remainder of the paper is organized as follows: - In Section II, we briefly describe the literature of Discrete Cosine Transform, Discrete Wavelet Transform and Singular Value Decomposition related to watermarking. Section III presents our proposed algorithm, while the simulations and data analysis are described in Section IV. Finally, we make some conclusions about our proposed method.

\section{LITERATURE REVIEW}

\section{A. Discrete Wavelet Transform}

The basic idea of discrete wavelet transform (DWT) in image process is to multi-differentiated decompose the image into sub-image of different spatial domain and independent frequency district. After the original image has been DWT transformed, the image is decomposed into four sub-band images by DWT: three high frequency parts (HL, LH and HH, named detail subimages) and one low frequency part (LL, named approximate sub-image). In Fig. 1, 2 level wavelet transform process of the image is shown, $\mathrm{HL}, \mathrm{LH}, \mathrm{HH}$ are the horizontal high frequency, the vertical high frequency and the diagonal high frequency part respectively and LL is the approximation low frequency part

The energy of the high-frequency part (horizontal, vertical and diagonal part) is less, which represent the information of 
the original image, such as the texture, edge, etc. The low frequency part concentrates most of the energy of the image and represents an important component and it can be decomposed continuously. The energy of the image is diffused better and the stronger image intensity can be embedded, with the more levels the image is decomposed by wavelet transform. Hence, the wavelet decomposing levels adopted in the algorithms can be chosen as far as possible.

\begin{tabular}{|c|c|}
\hline LL & HL \\
\hline LH & HH \\
\hline \multicolumn{2}{|c|}{ Fig. 1. Wavelet decomposition. } \\
\hline
\end{tabular}

\section{B. Discrete Cosine Transform}

The Discrete Cosine Transform is a very popular transform function that transforms a signal from spatial domain to frequency domain and it has been used in JPEG standard for image compression due to good performance. As a real transform, DCT transforms real data into real spectrum and therefore avoids the problem of redundancy. The popular block-based DCT transform segments an image non-overlapping block and applies DCT to each block. This result in giving three frequency sub-bands: low frequency sub band, mid-frequency sub-band and high frequency sub-band. DCT-based watermarking is based on two main facts. The first one is that most of the signal energy lies at low-frequencies sub band which contains the most important parts of the image and second one is that high frequency components of the image are usually removed through compression and noise attacks [19].

There are four established types of DCT's, i.e., DCT-I, DCT-II, DCT-III, and DCT-IV. The DCT-II is widely applied in signal processing because it is asymptotically equivalent to the Karhunen-Loeve Transform (KLT) for Markov-1 signals with a correlation coefficient that is close to one [20]. For example, JPEG image compression is also based on the DCT-II [21]. The two-dimensional DCT is usually used in digital image processing. Given an image $A$ of size $N \times N$, the DCT of the image is defined as:

$$
\begin{aligned}
& C(u, v)=\alpha(u) \alpha(v) \sum_{x=0}^{M-1} \sum_{y=0}^{N-1} f(x, y) \times \\
& \cos \left[\frac{\Pi(2 x+1) u}{2 M}\right] \cos \left[\frac{\Pi(2 y+1) v}{2 N}\right]
\end{aligned}
$$

And the inverse transform is defined as

$$
\begin{gathered}
f(x, y)=\sum_{u=0}^{M-1} \sum_{v=0}^{N-1} \alpha(u) \alpha(v) C(u, v) \times \\
\cos \left[\frac{\Pi(2 x+1) u}{2 M}\right\rceil \cos \left\lceil\frac{\Pi(2 y+1) v}{2 N}\right]
\end{gathered}
$$

where

$$
\alpha(u)=\left\{\begin{array}{l}
\frac{1}{\sqrt{M}}, u=0 \\
\sqrt{\frac{2}{M}}, u=1,2, \ldots \ldots, M-1
\end{array}\right.
$$

$$
\alpha(v)=\left\{\begin{array}{l}
\frac{1}{\sqrt{N}}, v=0 \\
\sqrt{\frac{2}{N}}, v=1,2, \ldots \ldots, N-1
\end{array}\right.
$$

\section{Singular Value Decomposition}

The singular value decomposition (SVD) is a factorization of a real or complex matrix, with many useful applications in signal processing and statistics.

The fundamental properties of SVD from the viewpoint of image processing applications are: i) the singular values (SVs) of an image have very good stability, i.e., when a small perturbation is added to an image, its SVs do not change significantly; and ii) SVs represent intrinsic algebraic image properties.

In this section, we describe a watermark casting and detection scheme based on the SVD.

From the viewpoint of linear algebra, we can observe that a discrete image is an array of nonnegative scalar entries, which may be regarded as a matrix. Let such an image be denoted by $A$. Without loss of generality, we assume in the subsequent discussions that $A$ is a square image, denoted by $A \in E^{N \times N}$, where $E$ represents either the real number domain or the complex number domain. The SVD of $A$ is defined as

$$
A=X \sum T^{H}
$$

where $X \in E^{N \times N}$ and $T \in E^{N \times N}$ are unitary matrices and $\sum \in E^{N \times N}$ is a diagonal matrix with nonnegative numbers on the diagonal and zeros on the off diagonal. The nonnegative components of $\Sigma$ represent the luminance value of the image. Changing them slightly does not change the image quality and they also don't change much even on attacks, watermarking algorithms normally make use of these two properties.

The unique property of the SVD transform is that the potential $N^{2}$ degrees of freedom (DOF) or samples in the original image now get mapped into

$$
\begin{gathered}
\sum \Rightarrow N \text { DOF } \\
X \Rightarrow \frac{N(N-1)}{2} \mathrm{DOF} \\
T \Rightarrow \frac{N(N-1)}{2} \mathrm{DOF} \\
\text { Totaling } N^{2} \mathrm{DOF}
\end{gathered}
$$

SVD has many good mathematical characteristics.

\section{PROposed MeTHOD}

This section presents the methods for embedding and extraction of hidden data. In this paper a DCT DWT SVD based blind watermarking technique has been used for embedding watermark. We use the DCT DWT SVD for host image and we select the middle frequency to embed watermark. 
The main task of this work has performed into following steps:

- Watermark Embedding

1) Apply one-level Haar DWT to decompose the host image A, into four sub-bands i.e. $A_{L L}, A_{H L}, A_{L H}$, and $A_{H H}$.

2) Consider $A_{H L}$ and is divided into $8 \times 8$ square blocks. Perform 2D DCT to each block, collect the DC value of each DCT coefficient matrix $D_{1}(x, y)$ together to get a new matrix $M_{1}$.

3) Now consider $A_{L H}$ and find the Coefficient matrix $D_{2}(x, y)$ and another new matrix $M_{2}$, same as step 2 .

4) Apply SVD to $M_{1}$ and $M_{2}$, obtain $M_{1}=U_{1} S_{1} V_{1}^{\mathrm{T}}$ and $M_{2}=U_{2} S_{2} V_{2}{ }^{\mathrm{T}}$.

5) Let $B$ of size $64 \times 64$ to represent the watermark image. Divide the $B$ into two parts: $B_{1}$ and $B_{2}$.

6) Modify the singular values $S_{1}$ and $S_{2}$ (in step 5) with $\mathrm{B}_{1}$ and $B_{2}$ respectively and apply $\mathrm{SVD}$ to them, $S_{1}+\alpha B_{1}=U_{1}{ }^{*} S_{1}{ }^{*} V_{1}{ }^{* *}$ and $S_{2}+\alpha B_{2}=U_{2}{ }^{*} S_{2}{ }^{*} V_{2}{ }^{T^{*}}$

7) For the coefficient matrix $D_{1}(x, y)$ in step 2 and $D_{2}(x, y)$ in step 3, change each DC value to $M_{1}{ }^{*}(x, y)$ and $M_{2}{ }^{*}(x, y)$, obtain new coefficient matrix $D_{1}{ }^{*}(x, y)$ and $D_{2}{ }^{*}(x, y)$ respectively. Apply inverse DCT to each $D_{1}{ }^{*}(x, y)$ and $D_{2}{ }^{*}(x, y)$ to produce the watermarked middle frequency band $A_{H L}{ }^{*}$ and $A_{L H}{ }^{*}$

8) The watermarked image, $A_{W}$ is obtained by performing the inverse DWT using two sets of modified DWT coefficient $\left(A_{H L}{ }^{*}\right.$ and $\left.\mathrm{A}_{\mathrm{LH}}{ }^{*}\right)$ and two sets of non-modified DWT coefficient $\left(A_{L L}\right.$ and $\left.A_{H H}\right)$.

- Watermark Extraction

1) Apply one-level Haar DWT to decompose the Watermarked image (possibly attack) $\mathrm{A}_{\mathrm{W}}$ into four sub-bands: $A_{L L}, A_{H L}{ }^{* *}, A_{L H}{ }^{* *}$, and $A_{H H}$.

2) Divide both of $A_{H L}{ }^{* *}$ and $A_{L H}{ }^{* *}$ into $8 \times 8$ square blocks separately, apply DCT to each block. Collect the DC value to get matrix $M_{1}{ }^{* *}$ for $A_{H L}{ }^{* *}$ and $M_{2}{ }^{* *}$ for $A_{L H}{ }^{* *}$.

3) Apply SVD to $M_{1}{ }^{* *}$ and $M_{2}{ }^{* *}$, i.e. $M_{1}{ }^{* *}=U_{1}{ }^{* * *} S_{1}{ }^{* *} V_{1}{ }^{T^{* *}}$ and $M_{2}{ }^{* *}=U_{2}{ }^{* *} S_{2}{ }^{* *} V_{2}{ }^{\mathrm{T}^{* *}}$.

4) Compute $C_{1}=U_{1}{ }^{*} S_{1}{ }^{* *} V_{1}{ }^{\mathrm{T} *}$ and $C_{2}=U_{2}{ }^{*} S_{2}{ }^{* * *} V_{2}{ }^{\mathrm{T} *}$

5) Extract the watermark image from each sub-band, i.e., $B_{1}{ }^{*}=\left(C_{1}-S_{1}\right) / \alpha$ and $B_{2}{ }^{*}=\left(C_{2}-S_{2}\right)$.

6) We get the watermark image by combining the results of step 5: $B^{*}=B_{1}{ }^{*}+B_{2}{ }^{*}$.

\section{SIMUlation AND ANALYSIS}

In order to testing the robustness of the proposed algorithm, substantial testing are performed. In the simulation, we test different manipulations on the four well-known and standard grayscale image that are "Lenna", "Boat", "Opera House" and "Pepper". The original images (host image) are shown in Fig. 2(a), Fig. 3(a), Fig. 4(a) and Fig. 5(a) and the watermarked are shown in Fig. 2(b), Fig. 3(b), Fig. 4(b) and Fig. 5(b) respectively. The watermark, as shown in Fig. 6(a), is used in our simulation. Simulation results show that the quality of watermarked image is promising.

To test and verify the robustness of our watermarking algorithm, the watermarked image is attacked by Gaussian Noise, Filtering, Rotation and Salt and Pepper. These are shown in Fig. 2-Fig. 5.
Perceptual quality of the watermarked image is measured by calculating PSNR between host and watermarked image, at the receiver side, watermark is extracted from the watermarked image. Extracted watermark is evaluated by measuring its correlation with the original watermark. The PSNR value is calculated at different gain factor, when the gain factor value is to be high the PSNR value of the image increases (Shown in Table I).

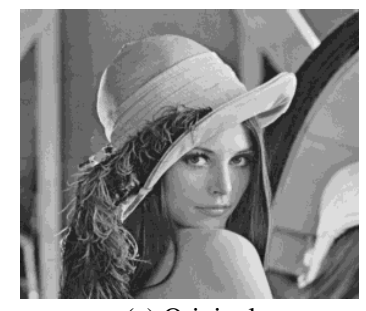

(a) Original

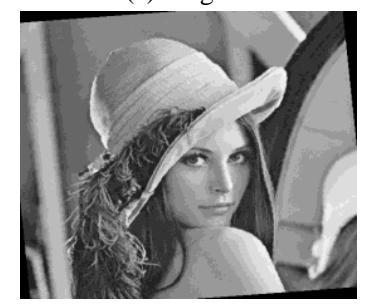

(c) Rotation

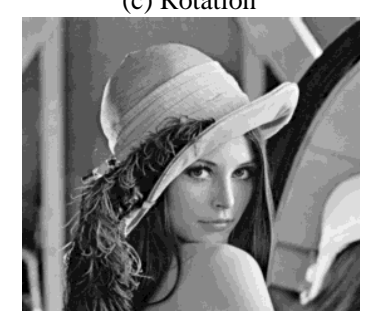

(e) Filtering

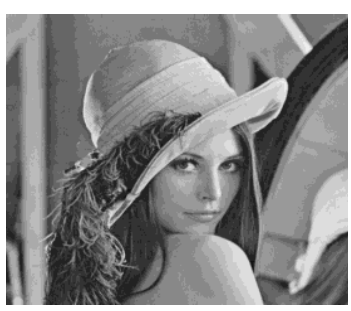

(b) Watermarked

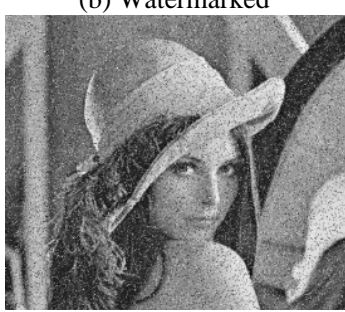

(d) Salt and pepper

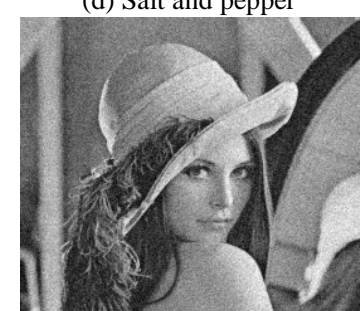

(f) Gaussian noise

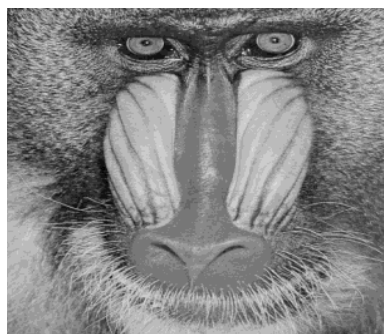

(a) Original

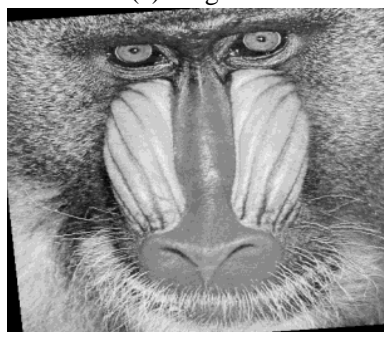

(c) Rotation

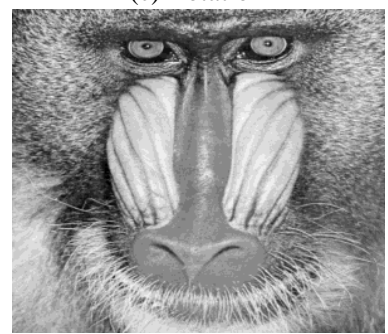

(e) Filtering

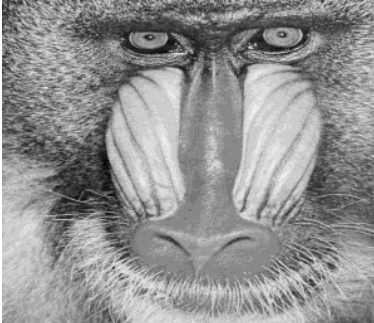

(b) Watermarked

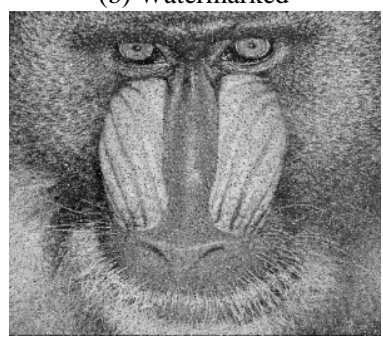

(d) Salt and pepper

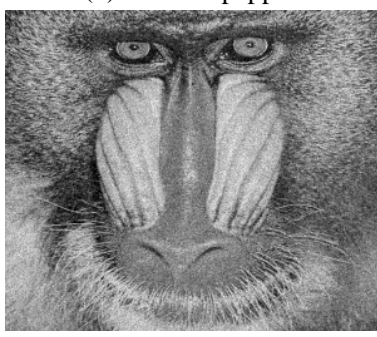

(f) Gaussian Noise
Fig. 3. Grayscale image "Baboon". 


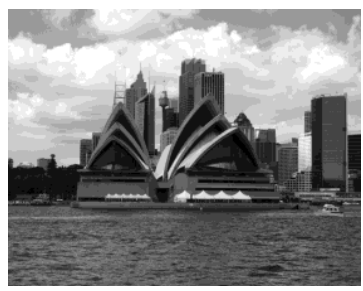

(a) Original

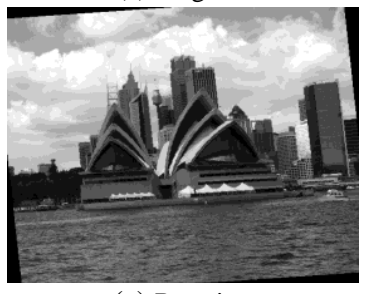

(c) Rotation

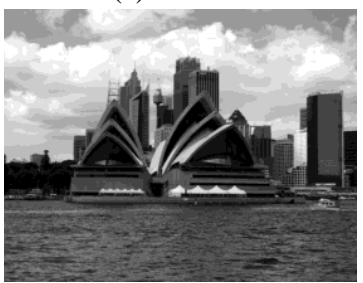

(e) Filtering

Fig. 4. Grayscale image "Opera House".

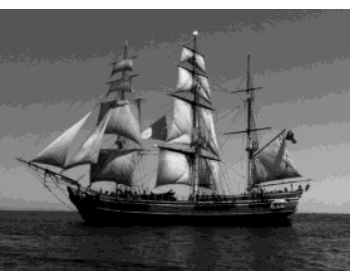

(a) Original

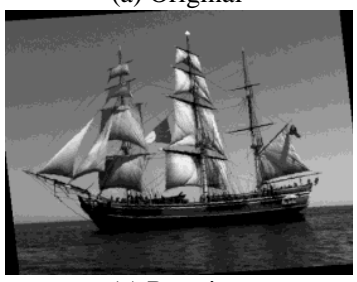

(c) Rotation

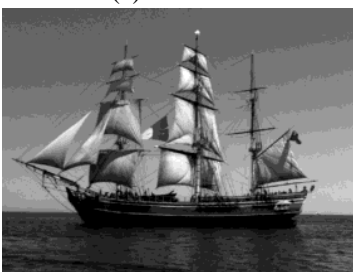

(e) Filtering

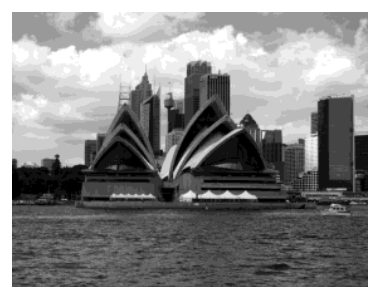

(b) Watermarked

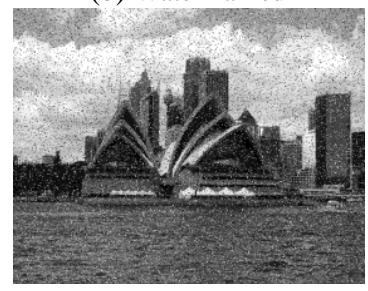

(d) Salt and pepper

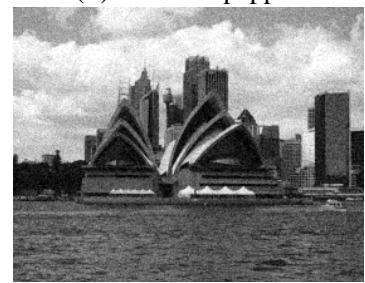

(f) Gaussian Noise

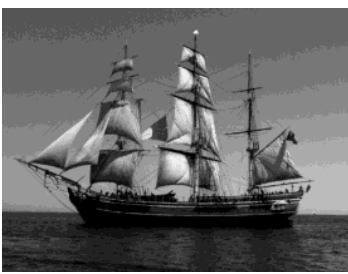

(b) Watermarked

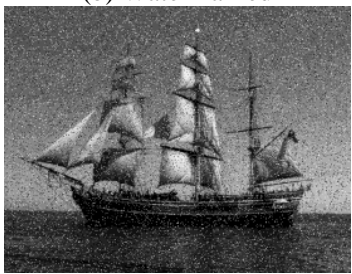

(d) Salt and pepper

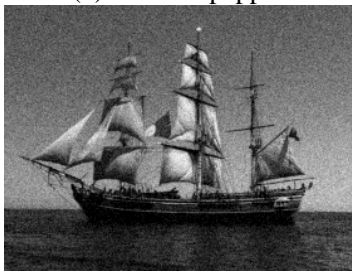

(f) Gaussian Noise

Fig. 5. Grayscale image "Boat".
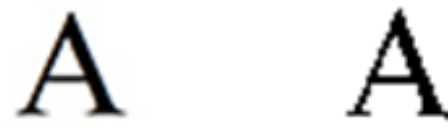

(a) Original

(b) Recovered

Fig. 6. Watermark image.

TABLE I: DIFFERENT VALUE OF PSNR FOR DIFFERENT IMAGES

\begin{tabular}{|c|c|}
\hline Image & PSNR(in dB) \\
\hline Lena & 51.318 \\
\hline Baboon & 51.209 \\
\hline Opera & 51.193 \\
\hline Boat & 50.998 \\
\hline
\end{tabular}

\section{CONCLUSION}

In this paper, a novel watermarking method based on DWT-DCT-SVD is proposed. This novel method gives successful results comparing to methods using different cover images. Results show that the new method is very robust against different attacks like Gaussian Noise, Salt and Pepper, filtering and Rotation. Therefore, the proposed algorithm is a good method for authentication of image materials.

\section{ACKNOWLEDGMENT}

This work is supported by University of Ulsan.

\section{REFERENCES}

[1] C. Rey and J. L. Dugelay, "A survey of watermarking algorithms for image authentication," Journal on Applied Signal Processing, vol. 2002, issue 6, pp. 613-621, 2002.

[2] G. Voyatzis, N. Nikolaidis, and I. Pitas, "Digital watermarking: an overview," in Proc. Ninth European Signal Processing Conference, Rhodes, Greece, September 8-11, 1998, pp. 9-12.

[3] V. Potdar, S. Han, and E. Chang, "A survey of digital image watermarking techniques," in Proc. 3rd IEEE-International Conference on Industrial Informatics, Frontier Technologies for the Future of Industry and Business, Perth, WA, August 10, 2005, pp. 709-716.

[4] N. J. Harish, B. B. S. Kumar, and A. Kusagur, "Hybrid robust watermarking technique based on DWT, DCT and SVD," International Journal of Advanced Electrical and Electronics Engineering, vol. 2, issue 5, 2013.

[5] J. Zhao, E. Koch, and C. Luo, "Digital watermarking in business today and tomorrow," Commun. ACM, vol. 41, no. 7, pp. 67-72, 1998.

[6] W. Zeng, "Digital watermarking and data hiding: technologies and applications," in Proc. ICISAS, 1998, vol. 3, pp. 223-229.

[7] S. Craver, N. Memon, B. L. Yeo, and M. M. Yeung, "Resolving rightful ownerships with invisible watermarking techniques: limitations, attacks and implications," IEEE J. Select. Areas Commun. vol. 16 , no. 4 , pp. 573-586, 1998.

[8] L. T. Qiao and K. Nahrstedt, "Watermarking schemes and protocols for protecting rightful ownership and customer's rights," J. Vis. Commun. Image Represent., vol. 9, no. 3, pp. 194-210, 1998.

[9] I. J. Cox, J. Kilian, F. T. Leighton, and T. Shamoon, "Secure spread spectrum watermarking for multimedia," IEEE Trans. Image Processing, vol. 6, pp. 1673-1687, Dec. 1997.

[10] N. Nikolaidis and I. Pitas, "Copyright protection of images using robust digital signatures," in Proc. ICASSP, May 1996, vol. 4, pp. 2168-2171

[11] N. Nikolaids and I. Pitas, "Robust image watermarking in the spatial domain," Signal Process., vol. 66, no. 3, pp. 385-403, 1998.

[12] M. Barni, F. Bartolini, V. Cappellini, and A. Piva, "A DCT-domain system for robust image watermarking," Signal Process., vol. 66, no. 3, pp. 357-372, 1998.

[13] L. Xie, G. R. Arce, A. Bash, and E. B. Basch, "Image enhancement toward soft image authentication," in Proc. IEEE International Conference on Multimedia and Expo (ICME), 2000, vol. 1, pp. 497-500.

[14] J. Fridirich, "Image watermarking for tamper detection," in Proc. IEEE International Conference on Image Processing (ICIP), 1998, vol. 2, pp. 404-408.

[15] C. Y. Lin and S. F. Chang, "Seme-fragile watermarking for authenticating JPEG visual content," in Proc. SPIE Int. Conf. Security and Watermarking of Multimedia Contents II, 2000, vol. 397, pp. 140-151

[16] M. Wu and B. Liu, "Watermarking for image authentication," in Proc. IEEE Int. Conf. Image Processing (ICIP), 2000, vol. 2, pp. 437-441.

[17] D. Kundur and D. Hatzinakos, "Digital watermarking for telltale tamper proofing and authentication," Proceedings of the IEEE, vol. 87, issue 7, pp. 1167-1180, July 1999.

[18] R. Sun, H. Sun, and T. Yao, "A SVD and quantization based semi-fragile watermarking for image authentication," in Proc. Int. Conf. Signal Processing (ICSP), 2002, vol. 2, pp. 26-30.

[19] C. C. Lai and C. C. Tsai, "Digital image watermarking using discrete wavelet transform and singular value decomposition," IEEE Transactions on Instrumentation and Measurement, vol. 59, no. 11, pp. 3060-3063, Nov. 2010. 
[20] K. R. Rao and P. Yip, Discrete Cosine Transform: Algorithms, Advantages, and Applications, Boston, MA: Academic, 1990.

[21] W. Pennebaker and J. Mitchell, JPEG Still Image Data Compression Standard, New York: Van Nostrand Reinhold, 1993.

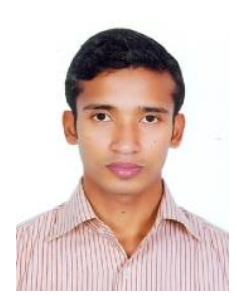

Md Saiful Islam is a lecturer of electrical and electronic engineering at Premier University, Bangladesh. He received B.Sc. degrees in electrical and electronic engineering from the Chittagong University of Engineering and Technology, Bangladesh in 2010.

Now, he is doing his M.Sc. in School of Electrical and Computer Engineering at University of Ulsan, South Korea and working as research assistant in digital signal processing and new media lab (DSPNM) at the same university. His research interests include digital signal processing, image processing, and green energy. He is currently researching the following advanced field: radar signal processing.

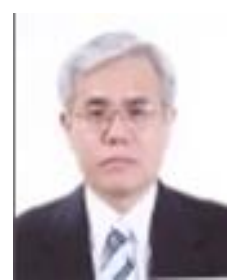

Ui Pil Chong received the B.S. degree in electrical engineering from University of Ulsan, Korea, in 1978, and M.S. degree in electrical engineering from Korea University, Seoul, Korea in 1980. He studied in field of computer engineering of Oregon State University and received M. S. degree in 1985 and received Ph. D. degree at New York University (POLY), NY, USA 1997.

In January of 1997, Dr. Chong joined the School of Computer Engineering and Information Technology of the University of Ulsan in Ulsan City, Korea where he has been promoted to be full professor since 2006. He has published more than 210 journal papers, conference papers in the area of digital signal processing, fault detection and diagnosis in the plants, biomedical engineering, computer music, and multimedia applications. He also holds the 10 Korean patents. He is a member of IEEE since 1993 and Eta Kappa Nu since 1995. He served as the president of Korean Society of Digital Arts Media from 2004 to 2007. Also he served as the dean of Graduate School of Information and Communication from 2004 to 2006 .

Currently, he is the head of Whale Research Institute in University of Ulsan and vice president of the Korea Institute of Signal Processing and Systems. 